\title{
The PARADIGHM (physicians advancing disease knowledge in hypoparathyroidism) registry for patients with chronic hypoparathyroidism: study protocol and interim baseline patient characteristics
}

Neil Gittoes ${ }^{1 *}$, Lars Rejnmark², Steven W. Ing ${ }^{3}$, Maria Luisa Brandi ${ }^{4}$, Sigridur Björnsdottir ${ }^{5}$, Stefanie Hahner ${ }^{6}$, Lorenz C. Hofbauer ${ }^{7}$, Pascal Houillier ${ }^{8}$, Aliya A. Khan ${ }^{9}$, Michael A. Levine ${ }^{10}$, Michael Mannstadt ${ }^{11}$,

Dolores M. Shoback ${ }^{12}$, Tamara J. Vokes ${ }^{13}$, Pinggao Zhang ${ }^{14}$, Claudio Marelli ${ }^{15}$, John Germak ${ }^{14}$ and Bart L. Clarke ${ }^{16}$

\begin{abstract}
Background: The PARADIGHM registry of adult and pediatric patients with chronic hypoparathyroidism evaluates the long-term safety and effectiveness of treatment with recombinant human parathyroid hormone, rhPTH(1-84), and describes the clinical disease course under conditions of routine clinical practice. In this first report, we detail the registry protocol and describe the baseline characteristics of two adult patient cohorts from an interim database analysis. One cohort after study entry were prescribed rhPTH(1-84), and the other cohort received conventional therapy of calcium and active vitamin D.

Methods: An observational study of patients with chronic hypoparathyroidism in North America and Europe, collecting data for $\geq 10$ years per patient. Main outcome measures were baseline patient demographics, clinical characteristics, medications, and disease outcome variables of symptoms, biochemical parameters, and health assessments. Baseline is the enrollment assessment for all variables except biochemical measurements in patients treated with rhPTH(1-84); those measurements were the most recent value before the first $\mathrm{rPPTH}(1-84)$ dose. Exclusion criteria applied to the analysis of specified outcomes included pediatric patients, patients who initiated rhPTH(1-84) prior to enrollment, and those who received rhPTH(1-34). Clinically implausible biochemical outlier data were excluded.
\end{abstract}

\footnotetext{
* Correspondence: Neil.Gittoes@uhb.nhs.uk

${ }^{1}$ Centre for Endocrinology, Diabetes and Metabolism (CEDAM), Queen

Elizabeth Hospital Edgbaston, 3rd Floor, Heritage Building, Birmingham B15

$2 \mathrm{TH}, \mathrm{UK}$

Full list of author information is available at the end of the article
}

(C) The Author(s). 2021 Open Access This article is licensed under a Creative Commons Attribution 4.0 International License, which permits use, sharing, adaptation, distribution and reproduction in any medium or format, as long as you give appropriate credit to the original author(s) and the source, provide a link to the Creative Commons licence, and indicate if changes were made. The images or other third party material in this article are included in the article's Creative Commons licence, unless indicated otherwise in a credit line to the material. If material is not included in the article's Creative Commons licence and your intended use is not permitted by statutory regulation or exceeds the permitted use, you will need to obtain permission directly from the copyright holder. To view a copy of this licence, visit http://creativecommons.org/licenses/by/4.0/ The Creative Commons Public Domain Dedication waiver (http://creativecommons.org/publicdomain/zero/1.0/) applies to the data made available in this article, unless otherwise stated in a credit line to the data. 
Results: As of 30 June 2019, data of 737 patients were analyzed from 64 centers; 587 (80\%) were women, mean \pm SD age $49.1 \pm 16.45$ years. At enrollment, symptoms reported for patients later prescribed $\mathrm{rhPTH}(1-84)(n=60)$ and those who received conventional therapy $(n=571)$, respectively, included fatigue $(51.7 \%, 40.1 \%)$, paresthesia $(51.7 \%, 29.6 \%)$, muscle twitching $(48.3 \%, 21.9 \%)$, and muscle cramping (41.7\%, 33.8\%). Mean serum total calcium, serum phosphate, creatinine, and estimated glomerular filtration rate were similar between cohorts. Health-related quality of life (HRQoL) 36-item Short Form Health Survey questionnaire scores for those later prescribed rhPTH(1-84) were generally lower than those for patients in the conventional therapy cohort.

Conclusions: At enrollment, based on symptoms and HRQoL, a greater percentage of patients subsequently prescribed rhPTH(1-84) appeared to have an increased burden of disease than those who received conventional therapy despite having normal biochemistry measurements. PARADIGHM will provide valuable real-world insights on the clinical course of hypoparathyroidism in patients treated with rhPTH(1-84) or conventional therapy in routine clinical practice.

Trial registration: EUPAS16927, NCT01922440

Keywords: Chronic hypoparathyroidism, parathyroid hormone, patient registry, quality of life, rhPTH(1-84), symptoms

\section{Introduction}

Hypoparathyroidism is a rare endocrine disorder resulting from undetectable or inappropriately low circulating levels of parathyroid hormone (PTH), which is the principal regulator of calcium and phosphate homeostasis $[1,2]$. The main biochemical abnormalities resulting from PTH deficiency are hypocalcemia and hyperphosphatemia [3]. The most common cause of hypoparathyroidism is neck surgery (approximately $75 \%$ of cases) $[4,5]$. Guidelines from the European Society of Endocrinology; the First International Conference on the Diagnosis, Management, and Treatment of Hypoparathyroidism; and the Canadian-led international working group provide recommendations for the diagnosis, treatment, and monitoring of chronic hypoparathyroidism in adults $[1,4,6]$. Patients with chronic hypoparathyroidism who are treated with conventional therapy consisting of calcium and active vitamin D supplements may still be inadequately controlled and have symptoms and risks of complications and comorbidities [3, 7-11]. In a 13-country patient and caregiver survey, the magnitude of symptom severity experienced by patients whose hypoparathyroidism was not adequately controlled despite receiving conventional therapy was associated with decreased health-related quality of life (HRQoL) and health status assessments and with increased caregiver burden [12]. Evaluation of HRQoL in patients with hypoparathyroidism has proved challenging because of variability of symptoms and differing types of study designs. Many but not all studies found there is a substantial burden of illness that adversely affects HRQoL in patients with chronic hypoparathyroidism [12-21]. A spectrum of symptomatology varying from no symptoms to severe symptoms was reported by patients with chronic hypoparathyroidism; in 36-item Short Form Health Survey questionnaire (SF-36) data transformed on a 0-100 scale (lower scores indicating poorer health states) the scores ranged from 20 to $>80$ in different domains [12].
The recombinant human parathyroid hormone (1-84), $\operatorname{rhPTH}(1-84)$, is full-length PTH that is approved in the United States and Europe as adjunctive treatment for patients with chronic hypoparathyroidism [22, 23]. In Europe, $\mathrm{rhPTH}(1-84)$ is indicated as adjunctive treatment for patients with chronic hypoparathyroidism who cannot be adequately controlled with conventional therapy alone. In the United States, $\operatorname{rhPTH}(1-84)$ is indicated as an adjunct to calcium and vitamin D to control hypocalcemia and is recommended only for patients who cannot be well controlled with calcium supplements and active vitamin D alone. The safety and efficacy of rhPTH(1-84) in patients with chronic hypoparathyroidism were first shown in short-term placebo-controlled clinical studies in which the need for conventional therapy was reduced, serum calcium levels were maintained, serum phosphate decreased, and events of hypocalcemia were reduced [24, 25]. In longer-term, open-label clinical studies, no new safety concerns were observed, reductions in oral calcium supplements and active vitamin D were achieved, serum calcium remained within target range, serum phosphorus and calcium-phosphate product levels improved, and urinary calcium excretion normalized [26-29].

Observational studies complement findings from interventional clinical trials and can provide real-world information on long-term outcomes of safety, effectiveness, and HRQoL across a broad population [30-32]. Patient registries, in particular for rare diseases, can obtain natural disease or treatment response data over the longterm that would otherwise be limited if performed by individual centers [31].

The multinational PARADIGHM (physicians advancing disease knowledge in hypoparathyroidism) registry (NCT01922440, EUPAS register number: EUPAS16927) is an opportunity to obtain valuable real-world data beyond those reported for clinical trials in patients with chronic hypoparathyroidism by providing long-term data 
for the treatment of patients with chronic hypoparathyroidism with rhPTH(1-84). PARADIGHM also records data that will allow for the assessment of the overall disease course in patients under conditions of routine clinical practice. Herein we describe the protocol for PARADIGHM and report baseline demographic and clinical characteristics for the current participants.

\section{Registry protocol \\ Design}

PARADIGHM is a prospective, observational registry designed to collect long-term data on patients with chronic hypoparathyroidism, whether they are receiving rhPTH(1-84) or conventional therapy, or both, under conditions of routine clinical practice. The registry data collection began in 2013, and enrollment is planned to close when an adequate number of patients have been enrolled to meet the study objectives. The original registry protocol was subsequently amended after $\mathrm{rhPTH}(1-$ 84) regulatory approval to include the designation as a non-interventional post-authorization safety study and to fulfill the post-marketing commitment request from the European Medicines Agency to the sponsor Shire, now a Takeda company. Data collection will continue for a minimum of ten years of follow-up for each patient; a final report is planned for 2035 or at the end of the product life cycle (ie, permanent cessation of release into the market/distribution chain) unless agreed otherwise with regulatory agencies.

\section{Objectives}

The protocol-defined primary objective of PARADIGHM is to characterize and describe the long-term safety and effectiveness of $\mathrm{rhPTH}(1-84)$ in patients with chronic hypoparathyroidism under conditions of routine clinical practice. The secondary objective is to characterize and describe the course of chronic hypoparathyroidism, including overall health status, patient-reported outcomes for symptoms of hypoparathyroidism, and healthcare resource utilization in patients treated or not treated with rhPTH(1-84) under conditions of routine clinical practice.

\section{Population}

The registry aims to enroll at least 900 patients with chronic hypoparathyroidism, including at least 300 patients receiving treatment with $\mathrm{rhPTH}(1-84)$ and at least 600 patients receiving conventional therapy alone (that includes calcium supplements and active vitamin D). All decisions regarding the management of chronic hypoparathyroidism for each patient are determined by the treating physician; no medications or recommendations are provided through participation in the registry. All adult patients (aged $\geq 18$ years) and pediatric patients (aged $<18$ years) with a diagnosis of chronic hypoparathyroidism (ie, hypoparathyroidism with a duration of $\geq 6$ months) receiving $\operatorname{rhPTH}(1-84)$ or conventional therapy are eligible for participation in the registry. Inclusion and exclusion criteria are listed in Table 1. The patient, the parent(s) of a pediatric patient, or a legally authorized representative is required to provide written informed consent. Patients may withdraw at any time for any reason without prejudice to their current or subsequent care. Patients who withdraw from the registry will be allowed to reenter, and any available data from the period the patient was not enrolled will be entered into the electronic case report form (eCRF). The study protocol is reviewed and approved by the Institutional Review Board (IRB)/Independent Ethics Committee/Research Ethics Board at each participating site or a centralized IRB service provider.

\section{Data collection}

The specific data to be entered into the registry database using the eCRFs at baseline and during subsequent followup clinic visits are listed in Table 2. Baseline is defined as the assessment at enrollment (visit 1) for all outcome variables except biochemical laboratory measurements in patients receiving treatment with rhPTH(1-84). The baseline for biochemical laboratory measurements was the most recent value before the first dose in patients receiving treatment with $\mathrm{rhPTH}(1-84)$. Patient data are to be entered into the registry database at intervals of at least every six months,

\section{Table 1 Criteria for registry participants}

Inclusion

- Patients with a diagnosis of chronic hypoparathyroidism and with a duration of $\geq 6$ months

- Adult patients aged $\geq 18$ years who are receiving conventional therapy, conventional therapy plus rhPTH(1-84), or rhPTH(1-84) alone

- Pediatric patients aged $<18$ years who are receiving conventional therapy, conventional therapy plus rhPTH(1-84), or rhPTH(1-84) alone

Exclusion

- Patients or legally authorized representatives unable to provide informed consent

- Patients using rhPTH(1-34), or used rhPTH(1-34) for $>2$ years and in the past 3 months

- Patients currently enrolled in an interventional clinical study whether or not the study is related to hypoparathyroidisma

- History of hypoparathyroidism resulting from a known activating mutation in the CASR gene

- History of hypoparathyroidism resulting from impaired responsiveness to PTH (pseudohypoparathyroidism)

PTH parathyroid hormone, $r$ PTH(1-34) recombinant human parathyroid hormone (1-34), rhPTH(1-84) recombinant human parathyroid hormone (1-84) ${ }^{a}$ Does not apply to those enrolled in other observational registries. The criteria were added during amendments to the original protocol to account for the inclusion of registry participants treated with $\mathrm{rhPTH}(1-84)$ prior to registry enrollment 
Table 2 Data collection schedule

\begin{tabular}{|c|c|c|}
\hline Parameter & Baseline visit & Follow-up visit \\
\hline Inclusion/exclusion criteria & $\times$ & \\
\hline Informed consent, medical records release, and contact order form & $\times$ & \\
\hline Demographics $^{\mathrm{a}}$ & $\times$ & \\
\hline Other study participation & $\times$ & $\times$ \\
\hline Hypoparathyroidism etiology (primary cause) ${ }^{b}$ & $\times$ & \\
\hline Family history & $x$ & \\
\hline Medical history/condition summary & $\times$ & \\
\hline Height and weight & $\times$ & $\times$ \\
\hline Pregnancy & $\times$ & $\times$ \\
\hline Clinical laboratory evaluations & $\times$ & $\times$ \\
\hline Other medical procedures ${ }^{c}$ & $\times$ & $\times$ \\
\hline Management of chronic hypoparathyroidism & $\times$ & $\times$ \\
\hline rhPTH(1-84) dosing information ${ }^{d}$ & $\times$ & $\times$ \\
\hline Historical PTH dosing information ${ }^{e}$ & $\times$ & \\
\hline Prior and concomitant medications, including over-the-counter medications & $\times$ & $\times$ \\
\hline Outcome evaluations (socioeconomic status and social history) & $\times$ & \\
\hline Outcome evaluations (Hypoparathyroidism Symptom Diary, WPAl:SHP, SF-36, SF-10) & $x^{f}$ & $\times$ \\
\hline Other questionnaires (signs and symptoms, hospitalization annual form) & $\times$ & $\times$ \\
\hline Vital status database searches & & $x^{9}$ \\
\hline Adverse events & $x^{f, h}$ & $\times$ \\
\hline Patient discontinuation & & $\times$ \\
\hline \multicolumn{3}{|c|}{ 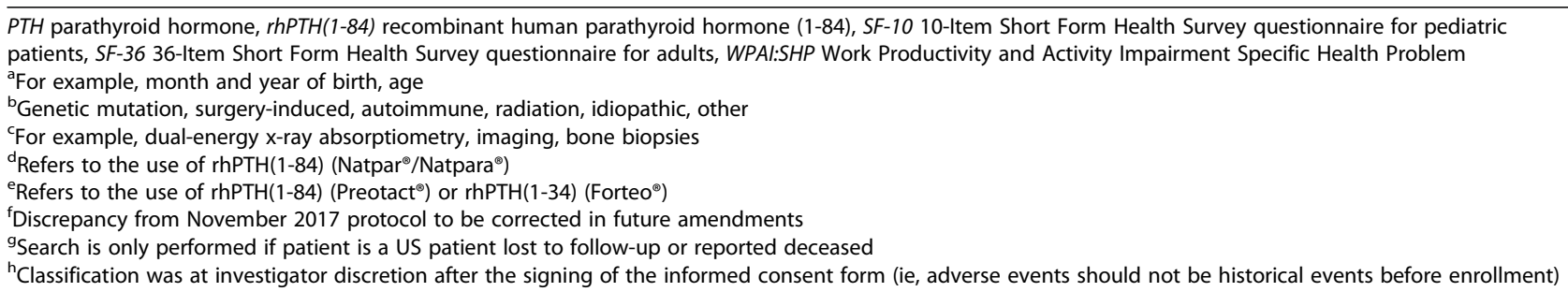 } \\
\hline
\end{tabular}

except for adverse events (AEs), which are collected on a continuous basis. The registry protocol AE definition is any pathologic, noxious, or unintended change in function indicated by changes in physical signs, symptoms, or laboratory evaluations. These include exacerbation of a preexisting condition and whether the $\mathrm{AE}$ is considered by the investigator as related to treatment with $\mathrm{rhPTH}(1-84)$ or not. Classification as an $\mathrm{AE}$ was at the investigator discretion after the patient signed the informed consent form, but AEs should not have been historical events prior to enrollment. AEs will be coded using the Medical Dictionary for Regulatory Activities (version 19.1 or later). The registry protocol serious $\mathrm{AE}$ (SAE) definition is any event that is life threatening or leads to death, requires inpatient hospitalization or prolongation of hospitalization, results in persistent or significant disability or incapacity, is a congenital abnormality or birth defect, or any other important medical event whether the SAE is considered by the investigator as related to treatment with rhPTH(1-84) or not.

\section{Outcome variables}

The primary outcome variables are AEs; clinical laboratory test results; biochemical laboratory test results for 24-hour urine calcium, serum total calcium, albumin-corrected serum calcium, magnesium and phosphate (measured as inorganic phosphate) serum levels, and $25[\mathrm{OH}] \mathrm{D}$; renal function assessments, including serum creatinine, creatinine-based estimated glomerular filtration rate (eGFR; analyzed in this report using the Chronic Kidney Disease Epidemiology Collaboration equation) [33], 24-hour urine calcium, and 24hour urine protein; renal calcification (eg nephrolithiasis, nephrocalcinosis); visits to an emergency department (ED) or hospitalization for renal events; presence of cataract; sites and numbers of bone fractures; and cardiovascular events. 
The secondary outcome variables include HRQoL evaluated using the Short Form Health Survey questionnaires (SF-36 for adult patients, SF-10 for pediatric patients), disease-specific patient-reported outcomes using the Hypoparathyroidism Symptom Diary [34, 35], chronic hypoparathyroidism-related visits to an ED or hospitalization, or other reasons for visits to an ED or hospitalization. In addition, the impact on daily life will be assessed using the Work Productivity and Activity Impairment Specific Health Problem questionnaire [36, 37].

\section{Statistical analysis}

No formal statistical testing is preplanned for registry data analyses. In general, categorical outcome variables are summarized as number of patients, percentage, and 95\% CI of patients in each category, and continuous outcome variables are summarized using descriptive statistics as number of patients, mean, SD, 95\% CI, and median (range).

\section{Interim registry database analysis: patient demographics and baseline characteristics}

This manuscript reports on the baseline patient demographic and clinical characteristics data collected for the overall analysis population and two adult patient cohorts predefined in the study design protocol: 1) patients prescribed $\mathrm{rhPTH}(1-84)$ after enrollment, and 2) patients receiving conventional therapy alone. Patients treated with rhPTH(1-34) were excluded, and patients who initiated treatment with $\mathrm{rhPTH}(1-84)$ prior to enrollment were excluded from specific analyses where this treatment could influence data reported at the time of enrollment (signs and symptoms of hypoparathyroidism reported within six months of enrollment; doctors' office visits, ED visits, and overnight hospital admissions due to hypoparathyroidism; and HRQoL SF-36 questionnaire). Analysis exclusions are noted within each relevant Table footer. In addition, biochemical parameters excluded patients with outlier data that after review were considered clinically implausible for patients with serum total calcium $<0.88 \mathrm{mmol} / \mathrm{L},>5.00 \mathrm{mmol} / \mathrm{L}(<3.5 \mathrm{mg} / \mathrm{dL},>20.0 \mathrm{mg} / \mathrm{dL})$; serum phosphate $<0.32 \mathrm{mmol} / \mathrm{L},>2.58 \mathrm{mmol} / \mathrm{L}(<1.0$ $\mathrm{mg} / \mathrm{dL},>8.0 \mathrm{mg} / \mathrm{dL}$ ); serum creatinine $<17.68 \mu \mathrm{mol} / \mathrm{L}$, $>884.00 \mu \mathrm{mol} / \mathrm{L}(<0.2 \mathrm{mg} / \mathrm{dL},>10.0 \mathrm{mg} / \mathrm{dL})$; or eGFR with negative values or values $>200.0 \mathrm{~mL} / \mathrm{min} / 1.73 \mathrm{~m}^{2}$.

The baseline data reported herein is for the following parameters: patient demographics; clinical characteristics; concomitant medications; disease-reported signs and symptoms; hypoparathyroidism-relevant biochemical parameters measured as part of routine clinical practice for serum total calcium, serum phosphate, serum creatinine and eGFR; the number of doctors' office visits, ED visits, and overnight hospital admissions in the 12 months before baseline; and HRQoL evaluated SF-36 questionnaires for adult patients.

As of 30 June 2019, the analysis population consisted of 737 patients (mean \pm SD age, $49.1 \pm 16.45$ years) from 64 centers in Europe and North America. The largest number of patients enrolled at a single center was 79 , two centers had enrolled 47 patients, four centers had enrolled between 20 and 40 patients, and 57 centers had enrolled $<20$ patients. The number of patients enrolled at each center is shown in Additional file 1. The study protocol was approved by each participating site via Independent Ethics Committees, Research Ethics Boards, or Institutional Review Boards (IRBs). For approvals by IRBs, either an institution-specific (27 US sites) or a centralized service provider who was able to review and approve for multiple sites (1 UK, 4 Swedish, and 19 US sites) were used. The Institutional Review Board of the Mayo Clinic, Rochester, Minnesota, USA approved the protocol for use at that institution.

The baseline patient demographics and clinical characteristics of the 737 participants are shown in Table 3. Most physicians treating patients with chronic hypoparathyroidism were endocrinologists $(n=660,89.6 \%)$. In adult patients, the most frequently used concomitant medications are shown in Table 4. Active vitamin D and analogs were prescribed for $90.2 \%$, calcium for $80.7 \%$, and thyroid hormones for $73.3 \%$ of adult patients. Antidepressants were prescribed more frequently for patients who were subsequently prescribed $\operatorname{rhPTH}(1-84)$ than in the conventional therapy cohort.

The most commonly reported signs and symptoms of hypoparathyroidism in adult patients within the previous six months of enrollment are shown in Table 5. At enrollment, muscle twitching and muscle cramping were reported in $48.3 \%$ and $41.7 \%$ of patients who were subsequently prescribed $\operatorname{rhPTH}(1-84)(n=60)$ and $21.9 \%$ and $33.8 \%$ of patients who received conventional therapy $(n=571)$, respectively. Fatigue and anxiety were reported in $51.7 \%$ and $26.7 \%$ of patients subsequently prescribed $\mathrm{rhPTH}(1-84)(n=60)$ and $40.1 \%$ and $20.7 \%$ of patients who received conventional therapy $(n=571)$, respectively.

Hypoparathyroidism-relevant biochemical parameters measured as part of routine clinical practice included serum total calcium, serum phosphate, serum creatinine, and eGFR and were similar in adult patients in both groups (Table 6). Adult patients who were subsequently prescribed $\mathrm{rhPTH}(1-84)$ showed a trend for higher numbers of doctors' office visits and ED visits in the 12 months before baseline versus those who received conventional therapy (Table 7). In addition, $45.0 \%$ of patients who were later prescribed $\mathrm{rhPTH}(1-84)$ and $14.5 \%$ of patients who received conventional therapy had $\geq 1$ overnight hospital admission over the same time frame (Table 7). 
Table 3 Baseline patient demographics and clinical characteristics

\begin{tabular}{|c|c|c|c|}
\hline Parameter & $\begin{array}{l}\operatorname{rhPTH}(1-84) \\
(n=134)\end{array}$ & $\begin{array}{l}\text { Conventional Therapy } \\
(n=603)\end{array}$ & $\begin{array}{l}\text { Analysis Population } \\
(N=737)\end{array}$ \\
\hline Age, mean $\pm S D, y$ & $47.9 \pm 14.22$ & $49.3 \pm 16.91$ & $49.1 \pm 16.45$ \\
\hline \multicolumn{4}{|l|}{ Age, category, n (\%), y } \\
\hline $0-17$ & $3(2.2)$ & $24(4.0)$ & $27(3.7)$ \\
\hline $18-39$ & $34(25.4)$ & $135(22.4)$ & $169(22.9)$ \\
\hline $40-64$ & $81(60.4)$ & $336(55.7)$ & $417(56.6)$ \\
\hline$\geq 65$ & $16(11.9)$ & $108(17.9)$ & $124(16.8)$ \\
\hline Women, n (\%) & $115(85.8)$ & $472(78.3)$ & $587(79.6)$ \\
\hline Race, white, n (\%) & $112(83.6)$ & $508(84.2)$ & $620(84.1)$ \\
\hline \multicolumn{4}{|l|}{$\mathrm{BMI}$, mean $\pm S D$, y } \\
\hline$<18^{\mathrm{b}}$ & $17.2 \pm 0.46$ & $19.6 \pm 6.07$ & $19.3 \pm 5.73$ \\
\hline$\geq 18^{c}$ & $31.1 \pm 9.48$ & $29.7 \pm 7.32$ & $30.0 \pm 7.72$ \\
\hline \multicolumn{4}{|l|}{ Primary cause of hypoparathyroidism, n (\%) } \\
\hline Surgery & $85(63.4)$ & $462(76.6)$ & $547(74.2)$ \\
\hline Idiopathic & $12(9.0)$ & $46(7.6)$ & $58(7.9)$ \\
\hline Genetic and autoimmune & $12(9.0)$ & $44(7.3)$ & $56(7.6)$ \\
\hline Radiation & 0 & $1(0.2)$ & $1(0.1)$ \\
\hline Unknown/other/missing & $25(18.7)$ & $50(8.3)$ & $75(10.2)$ \\
\hline \multicolumn{4}{|l|}{ Primary cause for thyroid surgery } \\
\hline Thyroid cancer, n (\%) & $37(56.1)$ & $244(60.7)$ & $281(60.0)$ \\
\hline Duration of hypoparathyroidism, mean $\pm S D$, $y^{d}$ & $9.0 \pm 10.27$ & $10.3 \pm 11.53$ & $10.1 \pm 11.34$ \\
\hline
\end{tabular}

$B M I$ body mass index, rhPTH(1-84) recombinant human parathyroid hormone (1-84)

${ }^{a}$ Baseline is defined as the assessment at enrollment (visit 1); 737 patients as of 30 June 2019

${ }^{b}$ rhPTH(1-84) $n=3$, conventional therapy $n=22$

crhPTH(1-84) $n=97$, conventional therapy $n=490$

${ }^{d}$ rhPTH(1-84) $n=110$, conventional therapy $n=563$

At baseline, the mean \pm SD SF-36 physical and mental component summary scores were $42 \pm 10.3$ and $42 \pm 12.8$ for patients in the subsequently treated $\mathrm{rhPTH}(1-84) \mathrm{co}-$ hort $(\mathrm{n}=60)$ and $46 \pm 10.4$ and $48 \pm 10.8$ for patients in the conventional therapy cohort $(\mathrm{n}=507)$, respectively. Reported outcome scores across individual SF-36 domains are illustrated in Table 8, which showed that scores at enrollment for patients subsequently treated with rhPTH(1-84) after enrollment trended lower in all domains than those who received conventional therapy.

\section{Registry and interim analysis limitations}

Information gathered from the large number of patients with chronic hypoparathyroidism enrolled in PARADIGHM will provide extensive outcome data relevant to routine clinical practice in patients treated with conventional therapy or rhPTH(1-84). However, because of the observational study design, there may be unobserved and/or inaccurate data that may limit generalized interpretation of the study outcomes. Because the PARADIGHM registry is ongoing, activity continues within participating centers to reconcile all missing data. In addition, no formal statistical comparisons between cohorts were planned, and the registry is not powered for hypothesis testing to compare outcome variables between the two cohorts. Furthermore, relatively few patients with nonsurgical etiologies for chronic hypoparathyroidism have enrolled in PARADIGHM; as a result, information collected in the registry may be most relevant to patients with post-surgical hypoparathyroidism. Finally, because of the low prevalence of this disease, some participating clinical centers have enrolled only one or a few patients into the registry. The registry may be representative of patient populations in Europe and North America, which have a large number of centers with enrolled patients, but a broader demographic and regional representation could be achieved with additional global (ie, Asia, Australia, Africa, and South America) participation of centers treating patients with chronic hypoparathyroidism.

\section{Discussion}

Because hypoparathyroidism is a rare disease, this multinational registry will enroll more patients with chronic hypoparathyroidism than is feasible for any single center or clinical trial. The design of the study protocol enables 
Table 4 Baseline concomitant medications used by $\geq 10 \%$ of adult patients ${ }^{a}$

\begin{tabular}{|c|c|c|c|}
\hline Medication, $\mathrm{n}(\%)^{\mathbf{b}}$ & $\begin{array}{l}\text { rhPTH(1-84) } \\
(n=125)\end{array}$ & $\begin{array}{l}\text { Conventional therapy } \\
(n=571)\end{array}$ & $\begin{array}{l}\text { Analysis population } \\
(N=696)\end{array}$ \\
\hline Vitamin $D$ and analogs & $105(84.0)$ & $523(91.6)$ & $628(90.2)$ \\
\hline Calcitriol & $86(68.8)$ & $430(75.3)$ & $516(74.1)$ \\
\hline Native vitamin D, including cholecalciferol and ergocalciferol & $75(60.0)$ & $255(44.7)$ & $330(47.4)$ \\
\hline Alfacalcidol & $1(0.8)$ & $55(9.6)$ & $56(8.0)$ \\
\hline Calcium & $96(76.8)$ & $466(81.6)$ & $562(80.7)$ \\
\hline Calcium carbonate & $62(49.6)$ & $333(58.3)$ & $395(56.8)$ \\
\hline Calcium citrate & $46(36.8)$ & $148(25.9)$ & $194(27.9)$ \\
\hline Calcium, not otherwise specified & $9(7.2)$ & $39(6.8)$ & $48(6.9)$ \\
\hline Thyroid hormone & $86(68.8)$ & $424(74.3)$ & $510(73.3)$ \\
\hline Levothyroxine & $79(63.2)$ & $417(73.0)$ & $496(71.3)$ \\
\hline Magnesium & $46(36.8)$ & $140(24.5)$ & $186(26.7)$ \\
\hline Proton pump inhibitors & $26(20.8)$ & $127(22.2)$ & $153(22.0)$ \\
\hline Benzodiazepine derivatives & $26(20.8)$ & $74(13.0)$ & $100(14.4)$ \\
\hline HMG-CoA reductase inhibitors & $16(12.8)$ & $115(20.1)$ & $131(18.8)$ \\
\hline Potassium & $25(20.0)$ & $66(11.6)$ & $91(13.1)$ \\
\hline Combinations of vitamins & $23(18.4)$ & $91(15.9)$ & $114(16.4)$ \\
\hline Antidepressants & $23(18.4)$ & $56(9.8)$ & $79(11.4)$ \\
\hline Selective serotonin reuptake inhibitors & $22(17.6)$ & $70(12.3)$ & $92(13.2)$ \\
\hline Platelet aggregation inhibitors ${ }^{d}$ & $10(8.0)$ & $92(16.1)$ & $102(14.7)$ \\
\hline Glucocorticoids & $17(13.6)$ & $91(15.9)$ & $108(15.5)$ \\
\hline Thiazides, hydrochlorothiazide & $16(12.8)$ & $76(13.3)$ & $92(13.2)$ \\
\hline Beta-blocking agents, selective & $15(12.0)$ & $67(11.7)$ & $82(11.8)$ \\
\hline
\end{tabular}

HMG-CoA reductase 3-hydroxy-3-methyl-glutaryl-coenzyme A reductase, $r$ PTH(1-84) recombinant human parathyroid hormone (1-84)

${ }^{\mathrm{a}}$ Drug class cutoff based on analysis population

${ }^{\mathrm{b}}$ Medication coded using the World Health Organization Drug Dictionary

'Baseline is defined as the assessment at enrollment (visit 1); pediatric patients ( $n=27)$ and those who received rhPTH(1-34) $(n=14)$ were excluded from the analysis

${ }^{\mathrm{d}}$ Excludes heparin

the collection of robust real-world data and informative disease-relevant parameters. It is expected that findings from PARADIGHM will increase the understanding of the course of hypoparathyroidism, provide insight into treatment effects in the patient populations, and stimulate further international effort and cooperation that may improve current clinical practices.

In this first report of the PARADIGHM registry, in addition to describing the protocol, we analyzed the baseline characteristics of the currently enrolled patient population. Despite serum calcium levels being maintained within the normal range, signs and symptoms of hypocalcemia, including paresthesia and muscle twitching, can persist in patients with chronic hypoparathyroidism that contribute toward the burden of illness, suggesting that the disease is not adequately controlled in these patients [9, 38]. At enrollment, the cohort of patients who were later prescribed $\operatorname{rhPTH}(1-84)$ showed a trend for increased reporting of symptoms related to chronic hypoparathyroidism, including paresthesia and muscle twitching, versus patients who received conventional therapy alone. Chronic hypoparathyroidism is associated with a range of physical, emotional, and mental health symptoms that impact the quality of life of patients, and which also impacts their caregivers [12, 14, 39]. At baseline, use of antidepressants was numerically higher in patients who were later prescribed $\operatorname{rhPTH}(1-$ 84) than in the conventional therapy cohort, and SF-36 scores across all domains were numerically lower for patients who were later prescribed $\operatorname{rhPTH}(1-84)$ than for patients who received conventional therapy. These data suggest that at baseline, patients enrolled in the rhPTH(1-84) group may have had a higher burden of symptoms than those in the conventional therapy group. The data presented in this publication predate any interruption of data collection due to the recall of $\mathrm{rhPTH}(1-$ $84)$ in the United States. Any patient who would discontinue $\mathrm{rhPTH}(1-84)$ treatment for any reason, including temporary product recall, is eligible for continued follow-up in the registry. Any change in therapy, from 
Table 5 Common symptoms reported within 6 months before enrollment ( $\geq 15 \%$ of adult patients in either cohort)

\begin{tabular}{llll}
\hline Parameter, $\boldsymbol{n}(\%)$ & $\begin{array}{l}\text { rhPTH(1-84) } \\
(\boldsymbol{n}=\mathbf{6 0})\end{array}$ & $\begin{array}{l}\text { Conventional therapy } \\
(\boldsymbol{n}=\mathbf{5 7 1})\end{array}$ & $\begin{array}{l}\text { Analysis population } \\
(\boldsymbol{N}=\mathbf{6 3 1}\end{array}$ \\
\hline Fatigue & $31(51.7)$ & $229(40.1)$ & $260(41.2)$ \\
Paresthesia & $31(51.7)$ & $169(29.6)$ & $200(31.7)$ \\
Muscle twitching & $29(48.3)$ & $125(21.9)$ & $154(24.4)$ \\
Muscle cramping & $25(41.7)$ & $193(33.8)$ & $218(34.5)$ \\
Headache & $20(33.3)$ & $105(18.4)$ & $125(19.8)$ \\
Muscle pain & $17(28.3)$ & $112(19.6)$ & $129(20.4)$ \\
Brain fog & $17(28.3)$ & $95(16.6)$ & $112(17.7)$ \\
Muscle weakness & $17(28.3)$ & $88(15.4)$ & $105(16.6)$ \\
Tetany & $17(28.3)$ & $71(12.4)$ & $88(13.9)$ \\
Joint pain & $16(26.7)$ & $138(24.2)$ & $154(24.4)$ \\
Anxiety & $16(26.7)$ & $118(20.7)$ & $134(21.2)$ \\
Weakness in extremities & $12(20.0)$ & $83(14.5)$ & $95(15.1)$ \\
Pain in extremities & $11(18.3)$ & $83(14.5)$ & $94(14.9)$ \\
Bone pain & $11(18.3)$ & $76(13.3)$ & $87(13.8)$ \\
Constipation & $11(18.3)$ & $72(12.6)$ & $83(13.2)$ \\
Nausea & $11(18.3)$ & $52(9.1)$ & $63(10.0)$ \\
Back pain & $9(15.0)$ & $106(18.6)$ & $115(18.2)$ \\
\hline
\end{tabular}

rhPTH(1-84) recombinant human parathyroid hormone (1-84)

The assessment at enrollment (visit 1) was defined as baseline; pediatric patients $(n=27)$, those who received rhPTH(1-34) $(n=14)$, and those who initiated rhPTH(1-84) before enrollment $(n=65)$ were excluded from the analysis

Table 6 Baseline biochemical parameters in adult patients

\begin{tabular}{|c|c|c|c|c|c|c|}
\hline \multirow[t]{2}{*}{ Parameter } & \multicolumn{2}{|c|}{$\begin{array}{l}\text { rhPTH(1-84) } \\
(n=125)\end{array}$} & \multicolumn{2}{|c|}{$\begin{array}{l}\text { Conventional therapy } \\
(n=571)\end{array}$} & \multicolumn{2}{|c|}{$\begin{array}{l}\text { Analysis population }{ }^{a} \\
(N=696)\end{array}$} \\
\hline & $\mathrm{n}$ & Value & $n$ & Value & $\mathrm{n}$ & Value \\
\hline \multicolumn{7}{|c|}{ Total serum calcium, mmol/L } \\
\hline Mean \pm SD & 83 & $2.2 \pm 0.34$ & 489 & $2.2 \pm 0.21$ & 572 & $2.2 \pm 0.24$ \\
\hline Median (range) & & $2.2(1.30-3.53)$ & & $2.2(1.15-3.15)$ & & $2.2(1.15-3.53)$ \\
\hline \multicolumn{7}{|c|}{ Serum phosphate ${ }^{\mathrm{b}}$, mmol/L } \\
\hline Mean \pm SD & 53 & $1.3 \pm 0.27$ & 280 & $1.4 \pm 0.26$ & 333 & $1.4 \pm 0.26$ \\
\hline Median (range) & & $1.3(0.8-1.9)$ & & $1.3(0.7-2.1)$ & & $1.3(0.7-2.1)$ \\
\hline \multicolumn{7}{|c|}{ Serum creatinine, $\mu \mathrm{mol} / \mathrm{L}$} \\
\hline Mean \pm SD & 69 & $86.9 \pm 55.93$ & 452 & $84.1 \pm 30.80$ & 521 & $84.5 \pm 35.11$ \\
\hline Median (range) & & $74.3(38.9-479.1)$ & & $78.3(26.5-284.6)$ & & $77.8(26.5-479.1)$ \\
\hline \multicolumn{7}{|c|}{$\mathrm{eGFR}, \mathrm{mL} / \mathrm{min} / 1.73 \mathrm{~m}^{2}$} \\
\hline Mean \pm SD & 69 & $83.2 \pm 23.73$ & 454 & $80.2 \pm 24.34$ & 523 & $80.6 \pm 24.25$ \\
\hline Median (range) & & $84.9(8.2-128.5)$ & & $81.1(0.5-162.5)$ & & $81.4(0.5-162.5)$ \\
\hline
\end{tabular}

eGFR estimated glomerular filtration rate, $r$ PTH(1-84) recombinant human parathyroid hormone (1-84)

${ }^{a}$ Baseline is defined as the assessment at enrollment (visit 1). The baseline measurement was the most recent value before the first dose in patients receiving treatment with $\mathrm{rPTH}(1-84)$; pediatric patients $(n=27)$, those who received $\mathrm{rhPTH}(1-34)(\mathrm{n}=14)$, and those with outlier data for total serum calcium $<0.88 \mathrm{mmol} / \mathrm{L}$ $>5.00 \mathrm{mmol} / \mathrm{L}(<3.5 \mathrm{mg} / \mathrm{dL},>20.0 \mathrm{mg} / \mathrm{dL}) ;$ serum phosphate $<0.32 \mathrm{mmol} / \mathrm{L},>2.58 \mathrm{mmol} / \mathrm{L}(<1.0 \mathrm{mg} / \mathrm{dL},>8.0 \mathrm{mg} / \mathrm{dL}) ;$ serum $\mathrm{creatinine}<17.68 \mu \mathrm{mol} / \mathrm{L},>884.00$ $\mu \mathrm{mol} / \mathrm{L}\left(<0.2 \mathrm{mg} / \mathrm{dL},>10.0 \mathrm{mg} / \mathrm{dL}\right.$ ); eGFR no lower limit/except negative values excluded, $>200.0 \mathrm{~mL} / \mathrm{min} / 1.73 \mathrm{~m}{ }^{2}$, were excluded from the analysis

${ }^{b}$ Inorganic phosphate was measured 
Table 7 Doctors' office visits, emergency department visits, and overnight hospital admissions due to hypoparathyroidism at baseline $^{a}$

\begin{tabular}{|c|c|c|c|}
\hline Parameter, n (\%) & $\begin{array}{l}\operatorname{rhPTH}(1-84) \\
(n=60)\end{array}$ & $\begin{array}{l}\text { Conventional therapy } \\
(n=571)\end{array}$ & $\begin{array}{l}\text { Analysis population }^{b} \\
(N=631)\end{array}$ \\
\hline \multicolumn{4}{|l|}{ Number of doctors' office visits } \\
\hline 0 & $8(13.3)$ & $123(21.5)$ & $131(20.8)$ \\
\hline 1 & $5(8.3)$ & $82(14.4)$ & $87(13.8)$ \\
\hline $2-3$ & $20(33.3)$ & $148(25.9)$ & $168(26.6)$ \\
\hline $4-5$ & $6(10.0)$ & $42(7.4)$ & $48(7.6)$ \\
\hline $6-7$ & $3(5.0)$ & $18(3.2)$ & $21(3.3)$ \\
\hline $8-9$ & $7(11.7)$ & $15(2.6)$ & $22(3.5)$ \\
\hline $10-12$ & $3(5.0)$ & $9(1.6)$ & $12(1.9)$ \\
\hline $13-15$ & $1(1.7)$ & $2(0.4)$ & $3(0.5)$ \\
\hline$\geq 16$ & $2(3.3)$ & $6(1.1)$ & $8(1.3)$ \\
\hline Missing & $5(8.3)$ & $126(22.1)$ & $131(20.8)$ \\
\hline \multicolumn{4}{|c|}{ Number of emergency department visits } \\
\hline 0 & $11(18.3)$ & $86(15.1)$ & $97(15.4)$ \\
\hline 1 & $15(25.0)$ & $41(7.2)$ & $56(8.9)$ \\
\hline $2-3$ & $5(8.3)$ & $25(4.4)$ & $30(4.8)$ \\
\hline $4-5$ & $3(5.0)$ & $6(1.1)$ & $9(1.4)$ \\
\hline $6-7$ & $3(5.0)$ & $8(1.4)$ & $11(1.7)$ \\
\hline $8-9$ & $0(0.0)$ & $0(0.0)$ & $0(0.0)$ \\
\hline $10-12$ & $1(1.7)$ & $1(0.2)$ & $2(0.3)$ \\
\hline $13-15$ & $0(0.0)$ & $1(0.2)$ & $1(0.2)$ \\
\hline$\geq 16$ & $0(0.0)$ & $0(0.0)$ & $0(0.0)$ \\
\hline Missing & $22(36.7)$ & $403(70.6)$ & $425(67.4)$ \\
\hline$\geq 1$ overnight hospital admission & $27(45.0)$ & $83(14.5)$ & $110(17.4)$ \\
\hline
\end{tabular}

rhPTH(1-84) recombinant human parathyroid hormone (1-84)

${ }^{a}$ Data are events reported in adult patients that occurred within 12 months before baseline

baseline is defined as the assessment at enrollment (visit 1); pediatric patients $(n=27)$, those who received rhPTH(1-34) $(n=14)$, and those who initiated rhPTH(1-

84) before enrollment $(n=65)$ were excluded from the analysis

Table 8 Baseline-reported outcome scores for SF-36 domains in adult patients

\begin{tabular}{|c|c|c|c|c|c|c|}
\hline \multirow[t]{2}{*}{ SF-36 Domain } & \multicolumn{2}{|c|}{$\begin{array}{l}\operatorname{rhPTH}(1-84) \\
(n=63)\end{array}$} & \multicolumn{2}{|c|}{$\begin{array}{l}\text { Conventional therapy } \\
(n=579)\end{array}$} & \multicolumn{2}{|c|}{$\begin{array}{l}\text { Analysis population }{ }^{a} \\
(N=642)\end{array}$} \\
\hline & $\mathrm{n}$ & Value & $\mathrm{n}$ & Value & $\mathrm{n}$ & Value \\
\hline Physical functioning & 62 & $43 \pm 11.6$ & 525 & $47 \pm 10.1$ & 587 & $47 \pm 10.3$ \\
\hline Bodily pain & 62 & $44 \pm 10.4$ & 530 & $47 \pm 10.8$ & 592 & $47 \pm 10.8$ \\
\hline Role limitation - physical health & 62 & $42 \pm 11.4$ & 527 & $46 \pm 11.0$ & 589 & $46 \pm 11.1$ \\
\hline Role limitation - emotional problems & 62 & $42 \pm 14.4$ & 530 & $47 \pm 11.1$ & 592 & $47 \pm 11.6$ \\
\hline General health & 61 & $41 \pm 10.4$ & 528 & $46 \pm 11.5$ & 589 & $45 \pm 11.5$ \\
\hline Mental health & 61 & $45 \pm 12.0$ & 529 & $49 \pm 10.2$ & 590 & $49 \pm 10.5$ \\
\hline Social functioning & 61 & $42 \pm 11.1$ & 530 & $47 \pm 10.9$ & 591 & $46 \pm 11.0$ \\
\hline Vitality & 61 & $42 \pm 11.5$ & 529 & $47 \pm 11.1$ & 590 & $47 \pm 11.2$ \\
\hline
\end{tabular}

All values are presented as mean \pm SD

rhPTH(1-84) recombinant human parathyroid hormone (1-84), SF-36 36-Item Short Form Health Survey questionnaire for adults

${ }^{a}$ Baseline is defined as the assessment at enrollment (visit 1); pediatric patients $(n=27)$ and those who initiated rhPTH(1-84) before enrollment ( $\left.n=68\right)$ were excluded from the analysis 
rhPTH(1-84) to conventional therapy or from conventional therapy to $\operatorname{rhPTH}(1-84)$, for any reason and at any time during the study is consistent with the registry protocol design and would be accounted for in data analysis according to the statistical analysis plan. Continuing assessment of biochemical and other parameters related to health status over the longer term will allow any differences in disease progression to be evaluated.

Information collected in PARADIGHM for patients with hypoparathyroidism encompasses areas of specific interest that are also included in other real-world registries, but there are a number of features that differentiate PARADIGHM from other national registries. The Danish National Patient Registry (DNPR) is a population-based administrative registry that has collected data from all Danish hospitals with complete coverage since 1978 [40]. An aim of the DNPR registry is to monitor the frequency of various diseases and treatments, whereas PARADIGHM collects detailed information on biochemical parameters and overall health status of patients with chronic hypoparathyroidism. The DNPR includes administrative data, diagnoses, treatment, and examinations that provide a sampling resource for longitudinal population-based clinical research. Patients with hypoparathyroidism in PARADIGHM had similar characteristics and etiology to those in the DNPR and Canadian National Hypoparathyroidism Registry (CNHR) that was formed in 2014. Although patient characteristics in PARADIGHM overall were similar to those in the CNHR, $81 \%$ of patients were reported to be receiving calcium and $74 \%$ were receiving calcitriol in PARADIGHM compared with 100\% receiving calcium supplements and $82.2 \%$ receiving calcitriol in the CNHR [41].

Despite the noted limitations, the real-world data gathered by PARADIGHM integrate patient demographics, clinical characteristics, biochemical data, and comorbidity data with symptom burden and impact of chronic hypoparathyroidism on quality of life that goes beyond that of other registries and clinical studies [12, 24-28, $42-45]$. The added value in the data will also come from the intended long follow-up period and data that will be captured for both adult and pediatric patients, including the opportunity to collect data for pediatric patients as they transition to adulthood.

In summary, PARADIGHM is expected to become a valued information resource about the clinical course of hypoparathyroidism, whether patients are treated with conventional therapy or rhPTH(1-84). PARADIGHM will also provide valuable longitudinal data on the safety and effectiveness of rhPTH(1-84) and may assist clinicians in the future management of patients with chronic hypoparathyroidism.

\section{Abbreviations}

AE: adverse event; CNHR: Canadian National Hypoparathyroidism Registry; DNPR: Danish National Patient Registry; eCRF: electronic case report form;
ED: emergency department; eGFR: estimated glomerular filtration rate; HRQOL: health-related quality of life; PARADIGHM: Physicians Advancing Disease Knowledge in Hypoparathyroidism; PTH: parathyroid hormone; rhPTH(1-84): recombinant human parathyroid hormone (1-84); SF-10: 10-item Short Form Health Survey questionnaire for pediatric patients; SF-36: 36-item Short Form Health Survey questionnaire for adults; WPAl:SHP: Work Productivity and Activity Impairment Specific Health Problem;

$1,25(\mathrm{OH})_{2} \mathrm{D}: 1,25$-dihydroxyvitamin D; 25(OH)D: 25-hydroxyvitamin D

\section{Supplementary Information}

The online version contains supplementary material available at https://doi. org/10.1186/s12902-021-00888-2.

Additional file 1: Figure S1. Number of patients in the analysis population of 737 patients enrolled at each of the 64 participating centers as of 30 June 2019.

\section{Acknowledgments}

We thank the extensive network of PARADIGHM registry site investigators and associated clinical staff. Under the direction of the authors, editorial support in the preparation of this manuscript was provided by Alan Storey, PhD (ICON, Marlow, Buckinghamshire, UK), and Sheila Curristin, PhD (ICON, North Wales, PA, USA)

\section{Authors' contributions}

MLB, BLC, JG, NG, PH, SH, SWI, AAK, MAL, CM, MM, LR, TV, and PZ participated in the conception and design of the work; SB, MLB, BLC, LCH, NG, PH, SH, SWI, AAK, MAL, MM, LR, DMS, and TV contributed to acquisition of the data; MLB, BLC, LCH, JG, NG, PH, SH, SWI, AAK, MAL, CM, MM, LR, TV, and PZ contributed to analysis of the data; SB, MLB, BLC, LCH, JG, NG, PH, SH, SWI, AAK, MAL, CM, MM, $L R$, DMS, TV, and PZ contributed to the interpretation of the data; $P Z$ performed statistical analyses. SB, MLB, BLC, LCH, JG, NG, PH, SH, SWI, AAK, MAL, CM, MM, $L R, D M S, T V$, and $P Z$ reviewed and revised the manuscript, agree to be fully accountable for ensuring the integrity and accuracy of the work, and read and approved the final manuscript.

\section{Funding}

The PARADIGHM registry and this data analysis were funded by Shire Human Genetic Therapies, Inc., a Takeda company, Lexington, MA, USA. Editorial support in the preparation of this manuscript was funded by Shire Human Genetic Therapies, a Takeda company, Lexington, MA, USA.

\section{Availability of data and materials}

The dataset supporting the conclusions of this article are included within the article. Restrictions apply to the availability of data generated or analyzed during this study to preserve patient confidentiality or because they were used under license. The corresponding author will on request detail the restrictions and any conditions under which access to some data may be provided.

\section{Declarations}

\section{Ethics approval and consent to participate}

The study protocol was reviewed and approved by the Institutional Review Board/Independent Ethics Committee/Research Ethics Board at each participating site. Central Institutional Review Boards were utilized by 1 UK, 4 Swedish, and 19 US sites; Institutional Review Boards were utilized by 27 US sites. The patient, the parent(s) of a pediatric patient, or a legally authorized representative is required to provide written informed consent. The study is being conducted in accordance with Good Pharmacoepidemiology Practice as described by the International Society for Pharmacoepidemiology.

\section{Consent for publication}

Not applicable

\section{Competing interests}

NG and SH have served as an advisory board member and consultant for Shire, a Takeda company. LR has served as an advisory board member, research investigator, and speaker for Shire, a Takeda company. SWI has 
served as an advisory board member and research investigator for Shire, a Takeda company, and served as research investigator for Amgen Inc., Chugai Pharmaceutical Co., Ltd, Radius Health Inc., and Ultragenyx Pharmaceutical. SB and AAK have served as research investigators for Shire, a Takeda company. MLB has served as a research investigator and speaker for Shire, a Takeda company. LCH has served as a research investigator and received honoraria for advisory boards from Shire, a Takeda company, and received honoraria for advisory boards from Ascendis Pharma, Alexion Pharmaceuticals Inc., Amgen Inc., Kyowa Kirin International, and USB. PH has served as an advisory board member, consultant, research investigator, and speaker for Shire, a Takeda company. MAL, DMS, and TV have served as an advisory board member, consultant, and research investigator for Shire, a Takeda company. PZ and JG are employed by Shire Human Genetic Therapies, Inc., a Takeda company, Lexington, MA, USA. MM has served as an advisory board member, consultant, and research investigator for Shire, a Takeda company, as consultant and research investigator for Chugai Pharmaceutical Co., Ltd, and as consultant for Amolyt Pharma. CM is employed by Takeda Pharmaceuticals International AG, Zurich, Switzerland. BLC has served as consultant and research investigator for Shire, a Takeda company, and has received grant funding from Ascendis Pharma and Chugai Pharmaceutical Co., Ltd.

\section{Author details}

${ }^{1}$ Centre for Endocrinology, Diabetes and Metabolism (CEDAM), Queen Elizabeth Hospital Edgbaston, 3rd Floor, Heritage Building, Birmingham B15 2TH, UK. ${ }^{2}$ Department of Endocrinology and Internal Medicine, Aarhus University Hospital, Aarhus, Palle Juul-Jensens Boulevard 99, 8200 Aarhus N, Denmark. ${ }^{3}$ Division of Endocrinology, Diabetes and Metabolism, 547 McCampbell Hall, Ohio State University Wexner Medical Center, 1581 Dodd Drive, Columbus, OH 43210, USA. ${ }^{4}$ Endocrinology and Metabolic Diseases, University of Florence, Viale Pieraccini 6, 50139 Florence, Italy. ${ }^{5}$ Department of Endocrinology, Metabolism, and Diabetes, Karolinska University Hospital Solna, SE-17176 Stockholm, Sweden. ${ }^{6}$ Department of Medicine I Endocrinology, and Diabetology, University Hospital Würzburg, Oberdürrbacher Str. 6, 97080 Würzburg, Germany. 'Division of Endocrinology, Diabetes, and Bone Diseases, Technische Universität Dresden Medical Center, Fetscherstrasse 74, D-01307 Dresden, Germany. ${ }^{8}$ Centre de Recherche des Cordeliers, INSERM, 15 Rue de l'Ecole de Médecine, Sorbonne Université, Université de Paris, Assistance Publique-Hôpitaux de Paris, 75006 Paris, France. ${ }^{9}$ Department of Medicine, McMaster University, 3075 Hospital Gate, Oakville, ON L6M 1M1, Canada. ${ }^{10}$ Division of Endocrinology and Diabetes, Children's Hospital of Philadelphia and University of Pennsylvania Perelman School of Medicine, 34th and Civic Center Boulevard, Philadelphia, PA 19104, USA. ${ }^{11}$ Endocrine Unit Massachusetts General Hospital and Harvard Medical School, 50 Blossom Street, Boston, MA 02114, USA. ${ }^{12}$ Endocrine Research Unit, San Francisco Department of Veterans Affairs Medical Center and University of California, 1700 Owens Street, San Francisco, CA 94158, USA. ${ }^{13}$ Section of Endocrinology, University of Chicago Medicine, 5841 South Maryland Avenue, Chicago, IL 60637, USA. ${ }^{14}$ Shire Human Genetic Therapies, Inc., a Takeda company, 45 Hayden Ave, Lexington, MA 02421, USA. ${ }^{15}$ Takeda Pharmaceuticals International AG, Thurgauerstrasse 130, 8152 Glattpark-Opfikon, Zurich, Switzerland. ${ }^{16}$ Division of Endocrinology, Diabetes, Metabolism, and Nutrition, Mayo Clinic, 1st Street SW, Rochester, MN, USA.

\section{Received: 19 November 2020 Accepted: 26 October 2021} Published online: 20 November 2021

\section{References}

1. Brandi ML, Bilezikian JP, Shoback D, Bouillon R, Clarke B, Thakker RV, et al. Management of hypoparathyroidism: summary statement and guidelines. J Clin Endocrinol Metab. 2016;101:2273-83.

2. Shoback D. Hypoparathyroidism. N Engl J Med. 2008;359:391-403.

3. Shoback DM, Bilezikian JP, Costa AG, Dempster D, Dralle H, Khan AA, et al. Presentation of hypoparathyroidism: etiologies and clinical features. J Clin Endocrinol Metab. 2016;101:2300-12.

4. Khan AA, Koch C, Van Uum SHM, Baillargeon JP, Bollerslev J, Brandi ML, et al. Standards of care for hypoparathyroidism in adults: a Canadian and international consensus. Eur J Endocrinol. 2019;180:P1-P22.

5. Powers J, Joy K, Ruscio A, Lagast H. Prevalence and incidence of hypoparathyroidism in the United States using a large claims database. J Bone Miner Res. 2013;28:2570-6.
6. Bollerslev J, Rejnmark L, Marcocci C, Shoback DM, Sitges-Serra A, van Biesen W, et al. European Society of Endocrinology clinical guideline: treatment of chronic hypoparathyroidism in adults. Eur J Endocrinol. 2015;173:G1-G120.

7. Mitchell DM, Regan S, Cooley MR, Lauter KB, Vrla MC, Becker CB, et al. Longterm follow-up of patients with hypoparathyroidism. J Clin Endocrinol Metab. 2012:97:4507-14.

8. Underbjerg L, Sikjaer T, Mosekilde L, Rejnmark L. Cardiovascular and renal complications to postsurgical hypoparathyroidism: a Danish nationwide controlled historic follow-up study. J Bone Miner Res. 2013;28:2277-85.

9. lqbal K, Dass N, Gip C, Vila J, Rylands AJ, Marelli C. Defining the characteristics of chronic hypoparathyroidism not adequately controlled on conventional therapy: consensus findings of three European delphi panels. Adv Ther. 2019;36:3007-16.

10. Meola A, Vignali E, Matrone A, Cetani F, Marcocci C. Efficacy and safety of long-term management of patients with chronic postsurgical hypoparathyroidism. J Endocrinol Invest. 2018;41:1221-6.

11. Tabacco G, Naciu AM, Maggi D, Santonati A, Pedone C, Cesareo R, et al. Cardiovascular autonomic neuropathy as a new complication of postsurgical chronic hypoparathyroidism. J Bone Miner Res. 2019;34:475-81.

12. Siggelkow H, Clarke BL, Germak J, Marelli C, Chen K, Dahl-Hansen H, et al. Burden of illness in not adequately controlled chronic hypoparathyroidism: findings from a 13-country patient and caregiver survey. Clin Endocrinol (Oxf). 2020;92:159-68.

13. Arlt W, Fremerey C, Callies F, Reincke M, Schneider P, Timmermann W, et al. Well-being, mood and calcium homeostasis in patients with hypoparathyroidism receiving standard treatment with calcium and vitamin D. Eur J Endocrinol. 2002;146:215-22.

14. Hadker N, Egan J, Sanders J, Lagast H, Clarke BL. Understanding the burden of illness associated with hypoparathyroidism reported among patients in the PARADOX study. Endocr Pract. 2014;20:671-9.

15. Astor MC, Lovas K, Debowska A, Eriksen EF, Evang JA, Fossum C, et al. Epidemiology and health-related quality of life in hypoparathyroidism in Norway. J Clin Endocrinol Metab. 2016;101:3045-53.

16. Cusano NE, Rubin MR, McMahon DJ, Irani D, Tulley A, Sliney J Jr, et al. The effect of PTH(1-84) on quality of life in hypoparathyroidism. J Clin Endocrinol Metab. 2013:98:2356-61.

17. Sikjaer T, Rolighed L, Hess A, Fuglsang-Frederiksen A, Mosekilde L, Rejnmark L. Effects of PTH(1-84) therapy on muscle function and quality of life in hypoparathyroidism: results from a randomized controlled trial. Osteoporos Int. 2014;25:1717-26.

18. Hepsen S, Akhanli P, Sakiz D, Sencar ME, Ucan B, Unsal IO, et al. The effects of patient and disease-related factors on the quality of life in patients with hypoparathyroidism. Arch Osteoporos. 2020;15:75.

19. Underbjerg $L$, Sikjaer T, Rejnmark L. Health-related quality of life in patients with nonsurgical hypoparathyroidism and pseudohypoparathyroidism. Clin Endocrinol (Oxf). 2018;88:838-47.

20. Cusano NE, Rubin MR, McMahon DJ, Irani D, Anderson L, Levy E, et al. $\mathrm{PTH}(1-84)$ is associated with improved quality of life in hypoparathyroidism through 5 years of therapy. J Clin Endocrinol Metab. 2014;99:3694-9.

21. Sikjaer T, Moser E, Rolighed L, Underbjerg L, Bislev LS, Mosekilde L, et al. Concurrent hypoparathyroidism is associated with impaired physical function and quality of life in hypothyroidism. J Bone Miner Res. 2016;31: 1440-8.

22. Natpar ${ }^{\oplus}$ parathyroid hormone. Dublin: Shire Pharmaceuticals Ireland Limited; 2019.

23. Natpara ${ }^{\circledast}$ parathyroid hormone. Lexington: Shire-NPS Pharmaceuticals, Inc:; 2020.

24. Mannstadt M, Clarke BL, Vokes T, Brandi ML, Ranganath L, Fraser WD, et al. Efficacy and safety of recombinant human parathyroid hormone (1-84) in hypoparathyroidism (REPLACE): a double-blind, placebo-controlled, randomized, phase 3 study. Lancet Diabetes Endocrinol. 2013;1:275-83 erratum: Lancet Diabetes Endocrinol. 2014;2(1):e3.

25. Sikjaer T, Rejnmark L, Rolighed L, Heickendorff L, Mosekilde L. Hypoparathyroid Study Group. The effect of adding PTH(1-84) to conventional treatment of hypoparathyroidism: a randomized, placebo-controlled study. J Bone Miner Res. 2011;26:2358-70.

26. Cusano NE, Rubin MR, McMahon DJ, Zhang C, Ives R, Tulley A, et al. Therapy of hypoparathyroidism with $\mathrm{PTH}(1-84)$ : a prospective four-year investigation of efficacy and safety. J Clin Endocrinol Metab. 2013;98:137-44.

27. Mannstadt M, Clarke BL, Bilezikian JP, Bone H, Denham D, Levine MA, et al. Safety and efficacy of 5 years of treatment with recombinant human 
parathyroid hormone in adults with hypoparathyroidism. J Clin Endocrinol Metab. 2019;104:5136-47.

28. Rubin MR, Cusano NE, Fan WW, Delgado Y, Zhang C, Costa AG, et al. Therapy of hypoparathyroidism with PTH(1-84): a prospective six year investigation of efficacy and safety. J Clin Endocrinol Metab. 2016;101:2742-50.

29. Tay YD, Tabacco G, Cusano NE, Williams J, Omeragic B, Majeed R, et al. Therapy of hypoparathyroidism with rhPTH(1-84): a prospective, 8-year investigation of efficacy and safety. J Clin Endocrinol Metab. 2019;104:5601-10.

30. Arne M, Janson C, Janson S, Boman G, Lindqvist U, Berne C, et al. Physical activity and quality of life in subjects with chronic disease: chronic obstructive pulmonary disease compared with rheumatoid arthritis and diabetes mellitus. Scand J Prim Health Care. 2009;27:141-7.

31. Mauro MJ, Davis C, Zyczynski T, Khoury HJ. The role of observational studies in optimizing the clinical management of chronic myeloid leukemia. Ther Adv Hematol. 2015;6:3-14.

32. Spigel DR. The value of observational cohort studies for cancer drugs. Biotechnol Healthc. 2010;7:18-24.

33. Levey AS, Stevens LA, Schmid CH, Zhang YL, Castro AF 3rd, Feldman HI, et al. A new equation to estimate glomerular filtration rate. Ann Intern Med. 2009;150:604-12.

34. Coles T, Chen K, Nelson L, Harris N, Vera-Llonch M, Krasner A, et al. Psychometric evaluation of the hypoparathyroidism symptom diary. Patient Relat Outcome Meas. 2019;10:25-36.

35. Martin S, Chen K, Harris N, Vera-Llonch M, Krasner A. Development of a patient-reported outcome measure for chronic hypoparathyroidism. Adv Ther. 2019;36:1999-2009.

36. Reilly Associates. Work productivity and activity impairment questionnaire: specific health problem v2.0 (WPAl:SHP). http://www.reillyassociates.net/ WPAl_SHP.html. Accessed Feb 2021.

37. Reilly MC, Zbrozek AS, Dukes EM. The validity and reproducibility of a work productivity and activity impairment instrument. Pharmacoeconomics. 1993; 4:353-65.

38. Hamdy NAT, Decallonne B, Evenepoel P, Gruson D, van VlokhovenVerhaegh L. Burden of illness in patients with chronic hypoparathyroidism not adequately controlled with conventional therapy: a Belgium and the Netherlands survey. J Endocrinol Invest. 2020. https://doi.org/10.1007/s4061 8-020-01442-y.

39. Vadiveloo T, Donnan PT, Leese CJ, Abraham KJ, Leese GP. Increased mortality and morbidity in patients with chronic hypoparathyroidism: a population-based study. Clin Endocrinol (Oxf). 2019;90:285-92.

40. Schmidt M, Schmidt SA, Sandegaard JL, Ehrenstein V, Pedersen L, Sorensen HT. The Danish National Patient Registry: a review of content, data quality, and research potential. Clin Epidemiol. 2015;7:449-90.

41. Alalawi Y, El Werfalli R, Abu Alrob H, Braga M, Millar A, Punthakee Z, et al. An overview of the etiology, clinical manifestations, management strategies, and complications of hypoparathyroidism from the Canadian National Hypoparathyroidism Registry. J Endocr Soc. 2019;3(Suppl 1):MON-LB1091. https://doi.org/10.1210/is.2019-MON-LB091.

42. Bilezikian JP, Clarke BL, Mannstadt M, Rothman J, Vokes T, Lee HM, et al. Safety and efficacy of recombinant human parathyroid hormone in adults with hypoparathyroidism randomly assigned to receive fixed $25-\mu \mathrm{g}$ or $50-$ $\mu \mathrm{g}$ daily doses. Clin Ther. 2017;39:2096-102.

43. Bohrer T, Paul J, Krannich H, Hasse C, Elert O. The Wuerzburg scale: a new classification system for permanent postoperative hypoparathyroidism. Eur Surg. 2007;39-44

44. Vokes TJ, Mannstadt M, Levine MA, Clarke BL, Lakatos P, Chen K, et al. Recombinant human parathyroid hormone effect on health-related quality of life in adults with chronic hypoparathyroidism. J Clin Endocrinol Metab. 2018;103:722-31.

45. Chen K, Krasner A, Li N, Xiang CQ, Totev T, Xie J. Clinical burden and healthcare resource utilization among patients with chronic hypoparathyroidism, overall and by adequately vs not adequately controlled disease: a multi-country chart review. J Med Econ. 2019:1-12.

\section{Publisher's Note}

Springer Nature remains neutral with regard to jurisdictional claims in published maps and institutional affiliations.

\section{Ready to submit your research? Choose BMC and benefit from:}

- fast, convenient online submission

- thorough peer review by experienced researchers in your field

- rapid publication on acceptance

- support for research data, including large and complex data types

- gold Open Access which fosters wider collaboration and increased citations

- maximum visibility for your research: over $100 \mathrm{M}$ website views per year

At BMC, research is always in progress.

Learn more biomedcentral.com/submissions 